\title{
Identification method of student card chip based on Internet of things radio frequency identification
}

\author{
XIANG Haiyun 1, FU Xiao 2
}

1. Modern Educational Technology Center, Southwest Petroleum University, Si Chuan, ChengDu, China

2. College of Computer Science, Southwest Petroleum University, Si Chuan, ChengDu, China

Key words: campus network; identity recognition; IC card; RFID

\begin{abstract}
The Thesis introduces an accurate location method facing campus student identity recognition on the basis of ultrahigh frequency RFID fingerprint. First, we obtain the fingerprint information in the sensor installed on the robot during the training stage, introduce the particle filter location algorithm on the basis of recurrence Bayes filter during the location stage and analyze the influence on location accuracy by measurement algorithm with different similarity during the correction stage; then, propose a new similarity measurement algorithm, Hsim, and conduct weight calculation on the prediction model combined with an observation model established by k-neighbor; finally, accurately estimate the robot's actual pose using re-sampling algorithm. Experiments have proven the location system's accuracy and effectiveness; besides, a precision of around $0.21539 \mathrm{~m}$ average location error is resulted. Experiment results show that the RFID fingerprint-based particle filter location system can preferably achieve overall location of mobile robot and that the system is presented with higher precision and robustness.
\end{abstract}

\section{Introduction}

Accurate and reliable location is a primary condition for automatic navigation of mobile robot. With the consistent expansion of the application domain of mobile robot and the progress of mobile robot navigation researches, navigation under non-structural environment, especially indoor unknown environment, has gradually become a hot problem in mobile robot territory. The RSS (Received Signal Strength) value of sign read by location fingerprint reader or their set with robot pose at current time. The RFID (Radio Frequency Identification) fingerprint information-based indoor mobile robot location method estimates the robot's location and direction angle at current time through comparing the similarity of currently-observed fingerprint and the reference one, combined with corresponding algorithm (prediction model).

\section{Overall realization method}

The system takes passive Ultra High Frequency label as the reference label. The robot is installed with a reader with two external antennas for obtaining the label's RSS. The particle filter location algorithm includes two stages: training stage for obtaining the environment's reference fingerprint information and location stage for completing mobile robot real-time pose calculation using the environment's fingerprint information. 


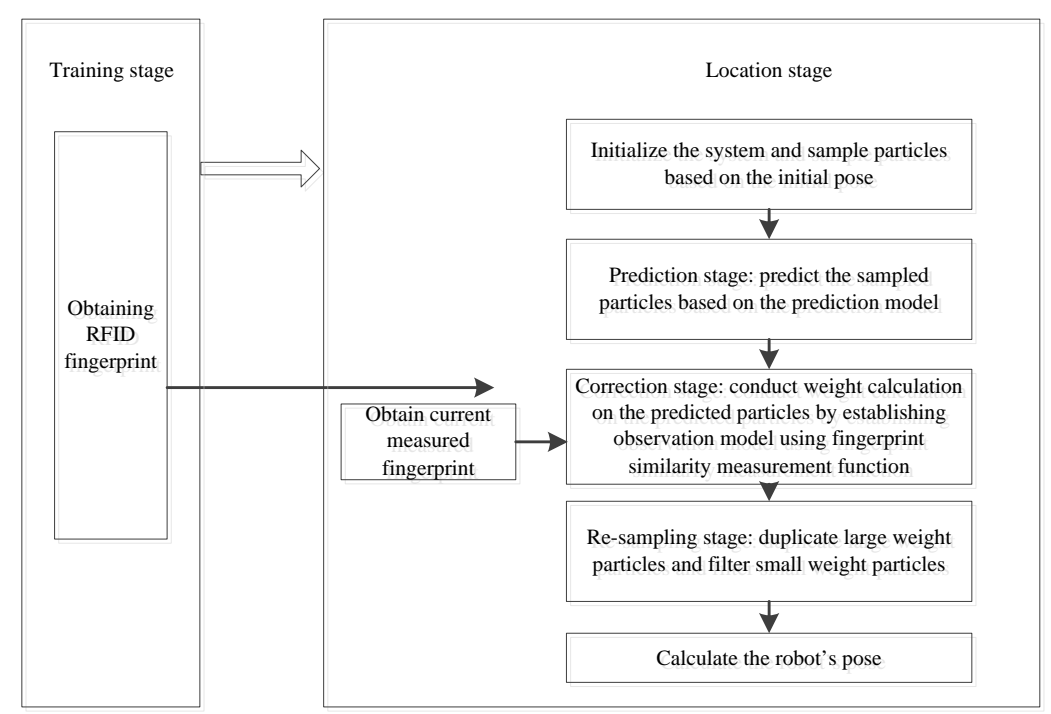

Fig. 1 RFID Fingerprint-based Particle Filter Location System

The overall realization method is as shown in Fig. 1. First, obtain the fingerprint information in the sensor installed on the robot (including the two-dimension coordinates of the robot's location and the robot's direction angle); then, introduce the particle filter location algorithm on the basis of recurrence Bayes filter during the location stage and conduct weight calculation on the prediction model by adopting the improved similarity measurement algorithm to establish an observation model; finally, accurately estimate the robot’s actual pose using re-sampling algorithm.

\section{RFID fingerprint recognition algorithm}

Particle filter has the advantages of high robustness and precision in mobile location territory and shows extraordinary performance in non-Gaussian and nonlinear system[7]. Approximate estimation of robot's pose at moment $t$ is realized using a series of randomly selected samples and such samples' significance weights. Robot pose estimation at moment $t$ can be expressed as the ith particle pose at moment t. Particle filter location algorithm can be divided into three steps: pre-estimation stage, correction stage and re-sampling stage.

a. Pre-estimation stage

As known that the mobile robot's pose at moment t-1 can be resulted from the posterior probability density of the robot at the previous moment, once a new mile-meter reading is given, the robot's pose at moment $t$ can be calculated using the pose at moment $\mathrm{t}-1$ and the robot's moving behavior. The established kinematics mobile robot prediction model is[8]:

Calculation of robot pose at moment $\mathrm{t}$ is as follows:

Where, is a random Gaussian noise whose mean value is 0 and variance is ; is the walking distance of the robot from t-1 to t; and is the change value of the robot's direction angle.

b. Correction stage

There is relatively large deviation for particles accepting particle status priori estimation prediction using system motion model during the pre-estimation stage, thus it is necessary to correct using current measurement values. On the premise of given reference fingerprint, the significance weight of each sampled particle's measurement value at moment $t$ is:

Normalization processing,

Where, is the observation model which will be specifically introduced next.

c. Re-sampling stage 
When the amount of small-weight particles surpasses certain limit, in order to avoid impoverishment of large-weight particles, which will further cause decreased location precision and even failure of providing location information, re-sampling algorithm is adopted to exclude the small-weight particles and replace them using large-weight particles. The Thesis introduces a refined re-sampling algorithm ER proposed by $\mathrm{Fu}$ et al[9] and Huang Baohu et al[10] to improve the particles refining capacities.

Make the setting that the system carries out re-sampling when the effective sample amount is smaller than half the total sample amount, calculation of re-sampling limit is as follows:

Particle filter realizes real-time overall location and tracking of robot through the above methods. The location of the robot can be expressed as:

\section{Similarity measurement algorithm}

Different high-dimension data and environment features remind us that the similarity measurement functions under different environments are not necessarily suitable for the system's working environment, thus it will be of significant influence on the location precision to select an appropriate similarity measurement algorithm. This section will study the similarity measurement methods for comparing the similar degree of two RFID fingerprint vectors and for particle weight calculation, analyze the performance of various similarity measurement functions being applied in high-dimension RFID fingerprint similarity measurement, and find out the similarity measurement function that is suitable for negative ultrahigh frequency RFID fingerprint location.

The similarity measurement methods quoted in this section and to be specifically introduced in the following text are as shown in Table 1. All functions are not negative, each function shows a reciprocity relationship with another. i.e. .

Table 1 Influence of Different Similarity Measurement Function sim on the Measurement of Label List f,g

\begin{tabular}{lll}
\hline \multicolumn{1}{c}{ Measurement function } & Abbreviation & Scope \\
\hline Cosine similarity & COS & {$[0,1]$} \\
Histogram intersection & HIST & {$[0, \infty)$} \\
Bhattacharyya coefficient & BHA & {$[0, \infty)$} \\
Hsim & Hsim & {$[0, \infty)$} \\
\hline Minkowski distance & $\mathrm{L}_{\mathrm{p}}$ & {$[0, \infty)$} \\
Hellinger distance & HD & {$[0, \infty)$} \\
$\chi^{2}$ statistics & CHI & {$[0, \infty)$} \\
Jeffrey divergence & JD & {$[0, \infty)$} \\
\hline Dot product & DOT & {$[0, \infty)$} \\
Number of common tags & NCT & {$[0, \infty)$} \\
\hline
\end{tabular}

Histogram intersection (HIST) measurement is a widely applied similarity measurement method that originates from the comparison of color histogram in image retrieval territory[12]. Similarity measurement result of two known fingerprint vectors using HIST means the shared accepted signal strength of the two fingerprint data. In the Thesis, it is assumed that the smaller RSS value is included in the larger one, thus the calculation value of similarity measurement result can somehow reflect the similarity degree of the two fingerprints.

Another classic similarity measurement function is Cosine that measures the similarity of two vectors using the cosine value of the angle formed by the two vectors. The features of this function lie in that the similarity is independent from vector amplitude, that it is especially good at disposing null value in high-dimension data, and that it is especially applicable to high-dimension RFID fingerprint data that include a large amount of empty RSS value. Among all the fingerprint data, at current moment, except a small part of labels are situated within the coverage area of RFID antenna, the other are unrecognizable, thus this part of accepted signal strength shall be taken as null. When measuring similarity using Cosine function, only when both fingerprints have value in the same dimension will the result contribute to similarity, the dot multiplication result will turn 0 as long as either of the two fingerprints is null. 


\section{Experimental verification}

Literature [5] proposes a Hsim similarity measurement algorithm and we are applying it to the particle filter of RFID fingerprint location in the Thesis. In the experiments, comparisons between classic similarity measurement algorithms, such as Cosine similarity, Histogram intersection and Bhattacharyya coefficient, and Hsim are made to show their performances in the Thesis. Besides, Root Mean Square Error of European distance is employed to measure the location effect. The experimental results are as shown in Fig. 2.

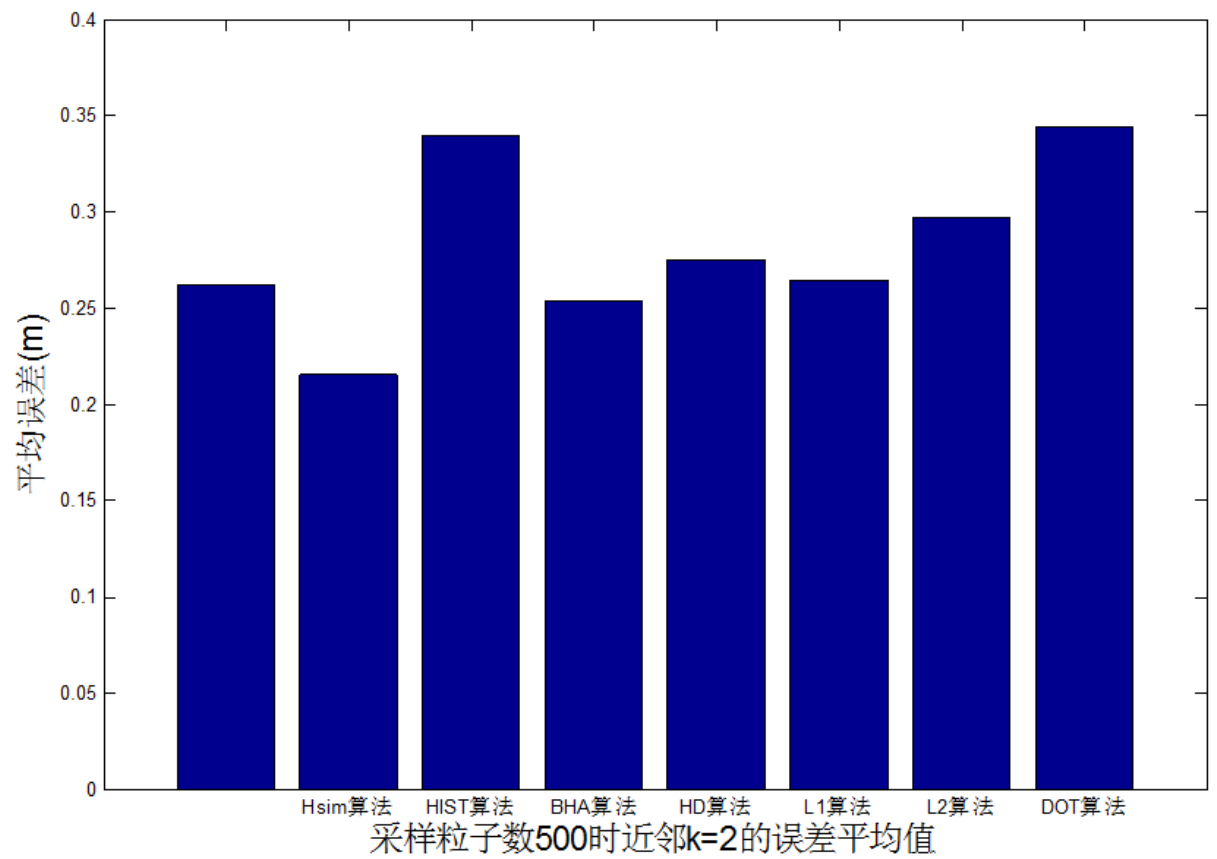

Fig. 2 Average System Errors Using Different Similarity Measurement Algorithms

Fig. 2 is average error histogram. Under the same experimental parameters, the average errors resulted from four re-sampling algorithms are 0.261756, 0.215390, 0.339858, 0.253390, 0.275449, $0.264805,0.297359,0.3445$ respectively (left $\rightarrow$ right), while the location precision of Hsim algorithm is $0.215390 \mathrm{~m}$, which is higher by $17.7 \%, 36.6 \%, 15.0 \%, 21.8 \%, 18.7 \%, 27.6 \%$ and $37.5 \%$ compared with other algorithms and 9.1\% higher than that of OSC algorithm proposed in Literature [1] (location precision $0.237 \mathrm{~m}$ ). Therefore, Hsim algorithm shows relatively large advantage among all similarity measurement algorithms.

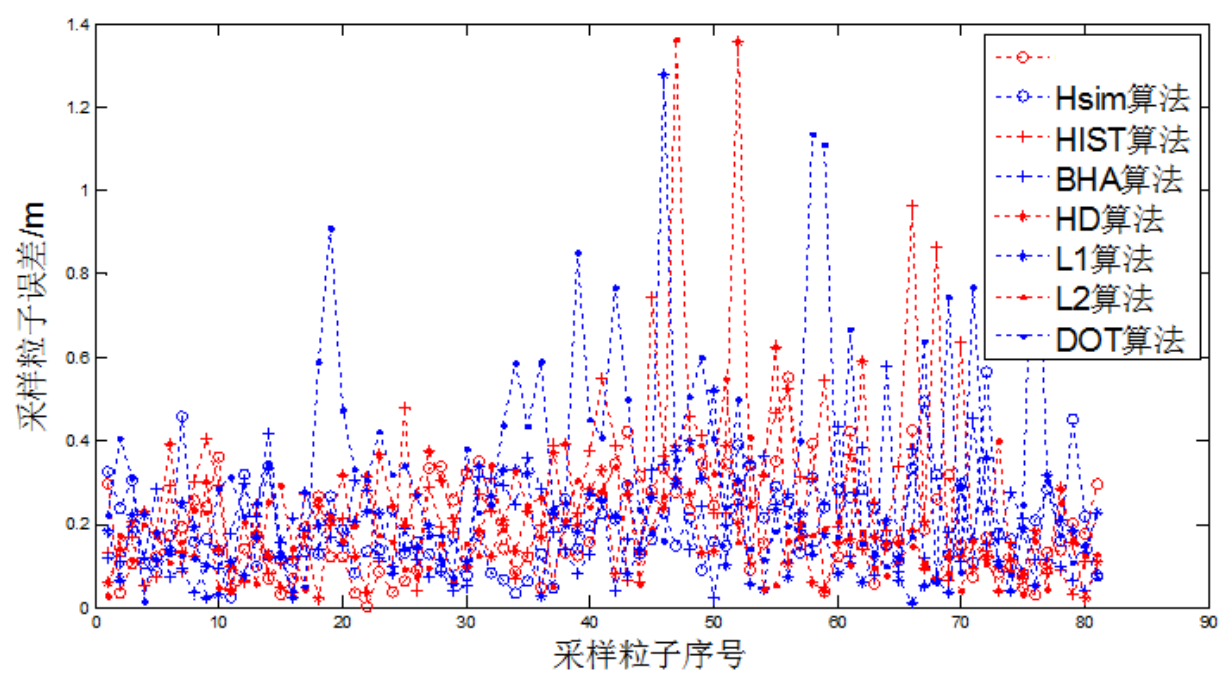

Fig. 3 System Errors Using Different Similarity Measurement Algorithms 
Sampling particle errors resulted from different similarity measurement algorithms are as shown in Fig. 3. Equal particle errors mean that the two particles share the same estimation pose while the smaller the particle deviation, the closer the sampling particles to the robot's actual pose. Both COS and Hsim algorithms get relatively low particle deviations, reflecting that they are equipped with good particle degeneracy resistance performances.

This section will present the influence of different sampling particle quantity in an algorithm on location precision. To make the experiment result analysis more convinced, straight corridor is selected as the experimental environment. Pasting adhesive tape on the floor to mark the robot's travel path, other experimental conditions keep consistent to the largest extent. Then, make the robot walk along the corridor for three times for each parameter. Obtain and analyze the average location error through multiple experiment results. When the quantities of sampling particles are $\mathrm{N}=10,50,100,500,1000,2000$, system operation results are as shown in Fig. 4:

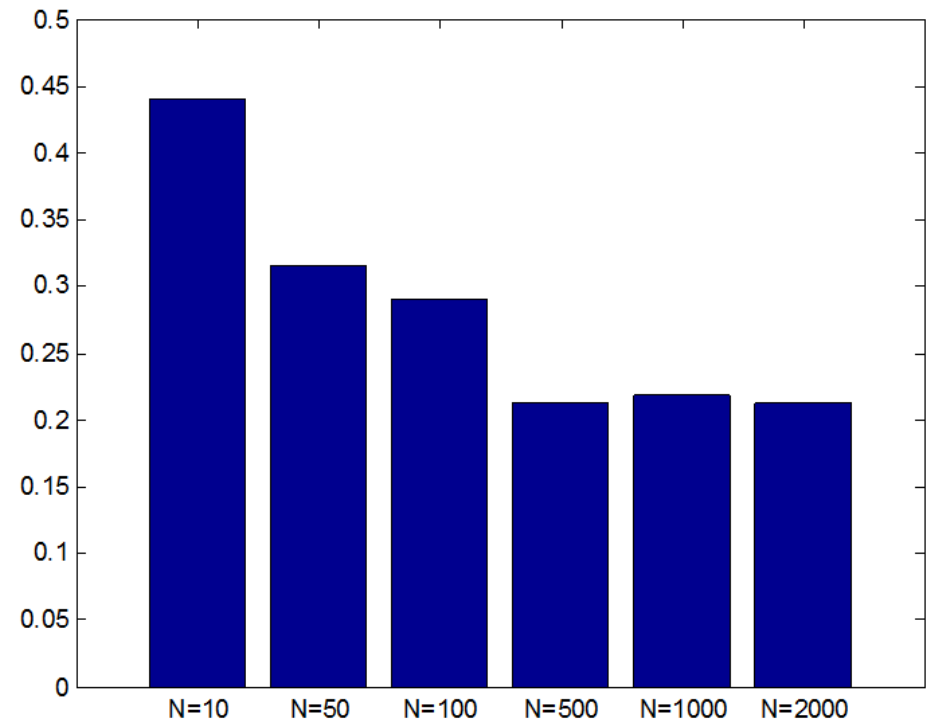

Fig. 4 System Errors under Different Sampling Particle Quantities

We can learn from the figure above that system location precision improves as the quantity of sampling particles keeps increasing. This is because the more the sampling particles, the more similar the location system to the actual estimated system location. However, when the sampling particle quantity increases from 500 to 2000, the location precision does not increase that much, while the system operation time rises as the quantity of sampling particles increases.

\section{Conclusion}

Aiming at traditional identity recognition fingerprint location's low precision and bad reliability, the Thesis studies particle filter fingerprint location methods with different similarity measurements and proposes a new negative RFID fingerprint self-location method according to vector space similarity measurement and k-neighbor weight algorithm (WKNN). During the training stage, obtain the fingerprint information by controlling the robot to mode within the location area; during the location stage, introduce the particle filter location algorithm on the basis of recurrence Bayes filter; then, propose a new similarity measurement algorithm, Hsim, and establish an observation model by analyzing the influence of different similarity measurement algorithm on location precision; finally, accurately estimate the robot's actual pose using re-sampling algorithm. After analyzing from different experimental aspects, it can be proven that the algorithm has significant improvement in both location precision and robustness.

\section{Reference}

[1] Lv, Z., Chirivella, J., \& Gagliardo, P. (2016). Bigdata Oriented Multimedia Mobile Health Applications. Journal of medical systems, 40(5), 1-10. 
[2] Lv, Z., Halawani, A., Feng, S., Li, H., \& Réhman, S. U. (2014). Multimodal hand and foot gesture interaction for handheld devices. ACM Transactions on Multimedia Computing, Communications, and Applications (TOMM), 11(1s), 10.

[3] Yang, J., Wang, H., Lv, Z., Wei, W., Song, H., Erol-Kantarci, M., ... \& He, S. (2016). Multimedia recommendation and transmission system based on cloud platform. Future Generation Computer Systems.

[4] Lv, Z., Halawani, A., Feng, S., Ur Réhman, S., \& Li, H. (2015). Touch-less interactive augmented reality game on vision-based wearable device. Personal and Ubiquitous Computing, 19(3-4), 551-567.

[5] Yang, J., Wang, Y., Li, B., Lu, W., Meng, Q., Lv, Z., ... \& Gao, Z. (2016). Quality Assessment Metric of Stereo Images Considering Cyclopean Integration and Visual Saliency. Information Sciences. 